\title{
High-capacity glycolytic and mitochondrial oxidative metabolisms mediate the growth ability of glioblastoma
}

\author{
JUNGIM KIM $^{1 *}$, JEONGSU HAN ${ }^{1 *}$, YUNSEON JANG ${ }^{1}$, SOO JEONG KIM ${ }^{1}$, MIN JOUNG LEE ${ }^{1}$, \\ MIN JEONG RYU ${ }^{1,2}$, GI RYANG KWEON ${ }^{2,4}$ and JUN YOUNG HEO ${ }^{1,3}$ \\ ${ }^{1}$ Department of Biochemistry, ${ }^{2}$ Research Institute for Medical Science, ${ }^{3}$ Brain Research Institute and \\ ${ }^{4}$ Infection Signaling Network Research Center, Chungnam National University School of Medicine, \\ Daejeon 301-747, Republic of Korea
}

Received May 23, 2015; Accepted July 6, 2015

DOI: $10.3892 /$ ijo.2015.3101

\begin{abstract}
Among the primary brain tumors, glioblastoma multiforme (GBM) has a radical proliferation ability that complicates the therapeutic modulation of cancer progression. The majority of GBM patients have a low survival rate $(<1$ year) due to radical tumor growth and late cancer diagnosis. Previous reports have shown that astrocytes have a specific metabolic organization that includes the production of lactate, the storage of glycogen, and use of lactate to support neurons which possess higher capacity of metabolism compared to neurons. We hypothesized that these characteristics of astrocytes could contribute to enhanced proliferation of GBM compared to neuroblastoma (NB). Here, we show that U87MG cells (a model of GBM) proliferate more rapidly than SH-SY5Y cells (a model of NB). A higher extracellular acidification rate and maximal mitochondrial oxygen consumption rate were observed in U87MG cells compared to SH-SY5Y cells. The expression levels of lactate dehydrogenase (LDH)-A and LDH-B were higher in U87MG cells and primary cultured astrocytes than in SH-SY5Y cells and neurons. Furthermore, the mRNA levels of succinate dehydrogenase and peroxisome proliferator-activated receptor- $\gamma$ were high in U87MG cells, suggesting that these cells have high capacity for mitochondrial metabolism and uptake of fatty acids related to synthesis of the cell membrane, respectively. Taken together, we demonstrate that GBM cells are characterized by activation of the LDH-expression-related glycolytic pathway and
\end{abstract}

Correspondence to: Professor Gi Ryang Kweon, Research Institute for Medical Science, Chungnam National University School of Medicine, Munhwa-dong, Jungu, Daejeon 301-747, Republic of Korea

E-mail: mitochondria@cnu.ac.kr

Dr Jun Young Heo, Department of Biochemistry, Chungnam National University School of Medicine, Munhwa-dong, Jungu, Daejeon 301-747, Republic of Korea

E-mail: junyoung3@gmail.com

${ }^{*}$ Contributed equally

Key words: glioblastoma multiforme, astrocyte, lactate dehydrogenase, oxygen consumption rate, extracellular acidification rate mitochondrial metabolic capacity, suggesting two innate properties of astrocytes that could provide a driving force for the growth ability of GBM. Based on these findings, we propose that therapeutic approaches aimed at treating GBM could target LDH for modulating the metabolic properties of GBM cells.

\section{Introduction}

Primary brain tumors have differential growth abilities that can affect the survival rates achieved by the current anticancer treatments. For example, oligodendroglioma is characterized by a slow growth rate and a prolonged median survival of 11.6 years for grade II disease and 3.5 years for grade III disease (1). Neuroblastoma (NB), which is most common in children, has a median survival of $\sim 27$ months for stage IV disease (2). The most common brain tumor, glioblastoma multiforme (GBM), is characterized by aggressive characteristics and rapid growth rate (3). The median survival of GBM patients is $\sim 1$ year despite the use of treatment strategies such as chemotherapy, radiotherapy and surgery (4). GBM is derived from astrocytes and has a poorer prognosis than other brain tumors due to its more rapid growth rate. We hypothesized that innate characteristics of astrocytes could be related to the progression of GBM.

In astrocytes, the metabolic flow of lipolysis and glycolysis is critical for the production of ATP and the maintenance of neuronal activity. Unlike neurons, astrocytes favor the production of lactate by aerobic glycolysis even in the absence of oxygen restriction (called the Warburg effect), while also efficiently producing ATP by mitochondrial respiration (called the Pasteur effect) (5). The metabolic capacity of astrocytes to produce lactate provides critical metabolic support to neurons (6). The transport of lactate through astrocytic monocarboxylate transporter (MCT) 4 has been shown to induce long-term potentiation and enhance learning and memory in the hippocampus (7). Primary cultured astrocytes isolated from mouse, release lactate to extracellular space (8) and L-lactate uptake to primary cultured neurons in embryonic rat (9). Pyruvate, produced from the oxidation of transported lactate from astrocytes acts as an energy source for mitochondrial ATP production in neurons. It is proved that pyruvate dehydroge- 
nase which convert from pyruvate to acetyl coenzyme A has shown strong immunoreactivity in neuronal cell body of the rat (10). Thus, the existing evidence suggests that lactate is involved in increasing growth by acting as an energy source, and supporting long-term memory formation by being shuttled between neurons and astrocytes. However, innate astrocytic metabolism could have a detrimental effect on the brain in the context of tumor formation, as the glycolytic and lipolytic fluxes in metabolism could support tumor cell proliferation.

Lactate dehydrogenase (LDH) catalyzes the interconversion between pyruvate and lactate. LDH-A, which prefer to catalyze the conversion of pyruvate to lactate, elevates the glycolytic rate in lactate-producing tissues (11). LDH-B, which prefer to catalyze the conversion of lactate to pyruvate, allows lactate to be used as an energy source for oxidative metabolism in aerobic tissues (11). LDH-A expresses primarily in astrocytes and LDH-B expresses in both astrocytes and neurons from hippocampus and occipital cortex of human (12). In tumor progression, high-level of LDH was reported in patients with GBM (13) and it associated with metastasis and survival in patients with brain tumor treated by radiotherapy (14). Thus, we hypothesized that the differential growth rate between GBM and NB are derived from high expression of inherent $\mathrm{LDH}$. The results of our study could contribute to the improving therapeutic strategies against GBM by clarifying the metabolic differences between astrocytes and neurons.

\section{Materials and methods}

Chemicals, reagents and antibodies. Oligomycin (O4876), carbonyl cyanide $m$-chlorophenyl hydrazone (CCCP, C2759) and rotenone (R8875) were purchased from Sigma-Aldrich (St. Louis, MO, USA). Trihydrochloride and TRIzol were purchased from Invitrogen (Camarillo, CA, USA). AntiLDH-A (rabbit polyclonal, NBP1-48336) and -LDH-B (rabbit monoclonal, EP1566Y) antibodies were purchased from Novus (Littleton, CO, USA). Anti-GFAP (rabbit polyclonal, ab7260) and -NeuN (mouse monoclonal, ab104224) antibodies were purchased from Abcam (Cambridge, MA, USA). The Cell Counting Kit-8 (CCK8) was purchased from Dojindo (Rockville, MD, USA).

\section{Cell culture}

Human glioblastoma U87MG cells and neuroblastoma SH-SY5Y cells. U87MG cells and SH-SY5Y cells were purchased from the Korean Cell Line Bank (Seoul National University, Seoul, Korea) and cultured in minimum essential medium (MEM; Welgene, Dalseo-gu, Daegu, Korea) supplemented with $10 \%$ fetal bovine serum (Invitrogen) and $1 \%$ penicillin/streptomycin (Invitrogen) at $37^{\circ} \mathrm{C}$ in a humidified atmosphere of $95 \%$ air and $5 \% \mathrm{CO}_{2}$.

Primary cortical astrocytes. Primary cortical astrocytes were prepared from postnatal 0-3 days P0-P3 C57BL/6 mice, as previously described (15). The cerebral cortex was dissected, freed from adherent meninges, and minced and triturated into a single-cell suspension. All experimental procedures were performed in accordance with the institutional guidelines of Chungnam National University School of Medicine (CNU, Daejeon, Korea). Cells were grown in Dulbecco's modified
Eagle's medium (DMEM, Invitrogen) supplemented with $25 \mathrm{mM}$ glucose, $10 \%$ heat-inactivated horse serum, 10\% heat-inactivated fetal bovine serum, $2 \mathrm{mM}$ glutamine and $1,000 \mathrm{U} / \mathrm{ml}$ penicillin-streptomycin. Cultures were maintained at $37^{\circ} \mathrm{C}$ in a humidified $5 \% \mathrm{CO}_{2}$ incubator. On the $3 \mathrm{rd}$ day of culture, cells were vigorously washed with repeated pipetting, and the medium was replaced. The next day (4th day of culture), cells were plated to coverslips $\left(1 \times 10^{4}\right.$ per coverslip) coated with $0.1 \mathrm{mg} / \mathrm{ml}$ poly-D-lysine (PDL).

Primary cortical neurons. The cerebral cortex was extracted from embryonic 14-15-day C57BL/6 mice, dispersed by trypsin and DNase I in Hank's balanced salt solution (HBSS), and then suspended in neurobasal media (Invitrogen) with 2\% B27 supplement, 2 mM L-glutamine and $1 \%$ penicillinstreptomycin. The cells were then filtered, transferred to 8-well culture dishes coated with polyethyleneimine $\left(1 \times 10^{5}\right.$ cells $\left./ \mathrm{ml}\right)$ in medium, and $1 \mu \mathrm{M}$ astrocyte inhibitor, cytosine-1- $\beta-\mathrm{D}$ arabinofuranoside (Sigma), treated after 2 days for purity of primary cultured neurons.

Measurement of cell growth rate. Cells were seeded to 96-well cell culture plates at $2 \times 10^{3}$ (for U87MG) and $8 \times 10^{3}$ (for SH-SY5Y) cells in $0.1 \mathrm{ml}$ growth media. Cell viability was measured after 12, 24, 48 and 72 h using a CCK-8 kit, a sensitive colorimetric assay for determining the number of viable cells. In this method, cellular dehydrogenases reduce WST- 8 to yield an orange-colored product (formazan) that is soluble in the tissue culture medium. The amount of generated formazan dye is directly proportional to the number of living cells. Absorbance was measured at $450 \mathrm{~nm}$ using a Multiskan Ascent microplate spectrophotometer (Thermo Fisher Scientific, Waltham, MA, USA).

Measurement of cellular oxygen consumption rate (OCR) and extracellular acidification rate (ECAR). OCR and ECAR were measured using a Seahorse Bioscience XF24 analyzer (Seahorse Bioscience, North Billerica, MA, USA). The XF24 biosensor cartridge was activated using $1 \mathrm{ml}$ of XF24 calibration buffer per well at $37^{\circ} \mathrm{C}$ with no $\mathrm{CO}_{2}$. U87MG and $\mathrm{SH}-\mathrm{SY} 5 Y$ cells were seeded to the XF24 cell culture microplates at $2 \times 10^{4}$ and $4 \times 10^{4}$ cells per well, respectively, in $0.1 \mathrm{ml}$ growth media. After 1 day, the medium was replaced with differentiation media, cells were incubated at $37^{\circ} \mathrm{C}$ with no $\mathrm{CO}_{2}$ for $\geq 1 \mathrm{~h}$, and measurements were performed. The ports of the XF24 biosensor cartridge were filled with $20 \mu \mathrm{g} / \mathrm{ml}$ oligomycin (an ATPase inhibitor), $50 \mu \mathrm{M}$ CCCP (an uncoupler) and $20 \mu \mathrm{M}$ rotenone (a mitochondrial complex I inhibitor) loaded together into each well, and the XF24 analyzer was operated under the manufacturer's basal protocol at $37^{\circ} \mathrm{C}$.

Real-time polymerase chain reaction $(P C R)$ analysis. Total RNA was isolated using TRIzol according to the manufacturer's instructions, and real-time quantitative PCR was performed using cDNA, SYBR Green PCR Master Mix (iCycleriQ Real-Time PCR Detection System; Bio-Rad, Hercules, CA, USA), and the following specific primers: $L D H-A$ F (5'-CTC TGA AGA CTC TGC ACC CA-3') and $L D H$-A R (5'-GCA CCC GCC TAA GAT TCT TC-3'); $P C$ F (5'-GCC TGG GAA GGT GAT AGA CA-3') and $P C$ R (5'-TCC AGT GTC ATG 
TCC TTG GT-3'); SDH F (5'-CAA CAC TCT AGC TTG CAC CC-3') and $S D H$ R (5'-GTA GAG CCC GTC CAG TTT CT-3'); and PPAR- $\gamma$ F (5'-TGC TTG TGA AGG ATG CAA GG-3') and PPAR- $\gamma$ R (5'-ATG AGA CAT CCC CAC TGC AA-3'). All primers were designed by the Primer3 program which is a widely used for designing PCR primers to amplify fragments that were appropriately sized $(<150 \mathrm{bp})$ for the Rotor-Gene 6000 real-time instrument (Qiagen, Valencia, CA, USA). Relative gene expression was quantified and normalized with respect to the 18s ribosomal RNA (housekeeping gene as endogenous control) using the Rotor-Gene 6000 real-time rotary analyzer software (Qiagen).

Western blot analysis. Proteins were extracted from U87MG cells, SH-SY5Y cells, primary cultured astrocytes and neurons with RIPA lysis buffer [100 mM Tris- $\mathrm{HCl}$ (pH 8.5), $200 \mathrm{mM}$ $\mathrm{NaCl}, 5 \mathrm{mM}$ EDTA and $0.2 \%$ SDS with phosphatase and a protease inhibitor cocktail]. Supernatants were centrifuged at $15,000 \mathrm{rpm}$ and $4^{\circ} \mathrm{C}$ for $20 \mathrm{~min}$, and protein levels were measured by the Bradford method. Isolated proteins $(10 \mu \mathrm{g})$ were resolved using 10\% SDS-PAGE and transferred onto polyvinylidene fluoride membranes, which were blocked with 5\% BSA in TBST [10 mM Tris- $\mathrm{HCl}$ (pH 7.6), $150 \mathrm{mM} \mathrm{NaCl}$, and $0.1 \%$ Tween-20]. The membranes were incubated overnight at $4^{\circ} \mathrm{C}$ with primary antibodies against $\operatorname{GFAP}(1: 10,000)$, NeuN $(1: 2,000)$, LDH-A $(1: 10,000)$, LDH-B $(1: 2,000)$ and $\alpha$-tubulin $(1: 10,000)$, and then with a horseradish peroxidasecoupled secondary antibody for $1 \mathrm{~h}$ at room temperature (RT). Finally, the antibody-labeled proteins were detected using an ECL system (INtRON BioTechnology, Seongnam, Gyeonggi, Korea).

Immunocytochemistry. U87MG and SH-SY5Y cells were seeded on poly-L-Lysine-coated coverslips at $2.5 \times 10^{4}$ cells per well in 12-well plates. The cells were fixed with $4 \%$ paraformaldehyde at room temperature (RT) for $15 \mathrm{~min}$, permeabilized with $0.02 \%$ BSA in PBS containing $0.25 \%$ Triton X-100 for 30 min, blocked with 5\% BSA in PBS at RT for $30 \mathrm{~min}$, and then incubated overnight at $4^{\circ} \mathrm{C}$ with primary antibodies against LDH-A (1:200), LDH-B (1:100), GFAP (1:400), or NeuN (1:300). The cells were then treated with Alexa Fluor 488-conjugated anti-chicken IgG, Alexa Fluor 594-conjugated anti-rabbit IgG and Alexa Fluor 647-conjugated anti-mouse IgG (Jackson ImmunoResearch, West Grove, PA, USA) at RT for $1 \mathrm{~h}$, and nuclei were stained with trihydrochloride (1:10,000). After each step, the cells were washed with $0.2 \%$ BSA in PBS. Finally, the cells were mounted on slides with fluorescent mounting medium (Dako North America, Carpinteria, CA, USA), and immunofluorescent images were acquired using an Olympus confocal microscope (Olympus, Center Valley, PA, USA).

Statistical analysis. The results are shown as mean values \pm SEM (error bars) and reflect at least three independent experiments. Statistical analyses included the 2-tailed unpaired Student's t-test and one-way analysis of variance (ANOVA), and were performed using GraphPad Instat software (GraphPad Software, San Diego, CA, USA). A P-value $<0.05$ was considered statistically significant, and such values are indicated in the figures by: ${ }^{*} \mathrm{P}<0.05,{ }^{* *} \mathrm{P}<0.01$ and ${ }^{* * *} \mathrm{P}<0.001$.

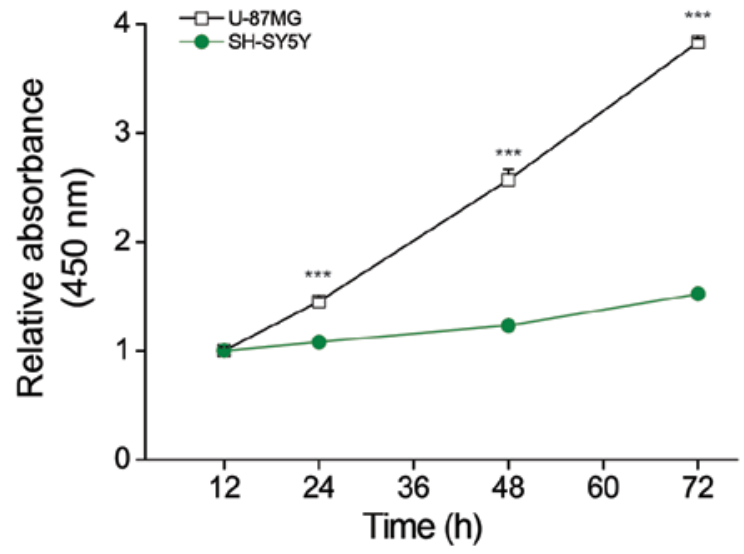

Figure 1. Cell growth over time in U87MG and SH-SY5Y cells. The cell growth rates of U87MG and SH-SY5Y cells were measured with a Cell Counting Kit-8 (CCK-8) from 12 to $72 \mathrm{~h}$. The data represent the mean \pm SEM (error bars) from three experiments (U87MG, $\mathrm{n}=12$ wells; and SH-SY5Y, $\mathrm{n}=12$ wells). ${ }^{* * *} \mathrm{P}<0.001$ (by one-way ANOVA) compared with SH-SY5Y cells.

\section{Results}

U87MG cells grow faster than SH-SY5Y cells. To examine the factors capable of affecting the growth rate of GBM, we first sought to characterize the growth rate of U87MG cells in a model system. We used the Cell Counting Kit-8 (CCK-8) assay to compare the proliferation ability of U87MG cells, which represent fast-growing GBM, and SH-SY5Y cells, which represent slower-growing NB (16) and were used herein as a control cancer cell line. As expected, we found that U87MG cells grew more rapidly ( $>2$-fold) than SH-SY5Y cells over a period of $48 \mathrm{~h}$ (Fig. 1).

U87MG cells show high-level of ECAR production but no difference in $O C R$. According to the Warburg effect, activation of aerobic glycolysis in cancer cells affects cell growth by supporting the production of ATP through the mitochondrial respiratory chain $(17,18)$. To compare mitochondrial function between U87MG and SH-SY5Y cells, we used an XF24 analyzer to evaluate the oxygen consumption rate (OCR) (Fig. 2A) and extracellular acidification rate (ECAR) (Fig. 2B) (19). Interestingly, there was no difference in the basal mitochondrial OCR (U87MG cells, $1.798 \pm 0.09 \mathrm{nmol}$ and SH-SY5Y cells, $2.025 \pm 0.077 \mathrm{nmol}$ ) (Fig. 2C). In contrast, ECAR was $\sim 3$-fold higher in U87MG cells compared to SH-SY5Y cells (U87MG cells, $0.407 \pm 0.025 \mathrm{nmol}$ and SH-SY5Y cells, $0.125 \pm 0.007 \mathrm{nmol}$ ) (Fig. 2D). Thus, the metabolic flow seems to favor aerobic glycolysis for ATP generation in U87MG cells. Following treatment of cells with carbonyl cyanide $\mathrm{m}$-chlorophenyl hydrazone (CCCP), which is potent mitochondrial oxidative phosphorylation uncoupler (20), U87MG cells were found to have a higher capacity for maximal OCR (U87MG cells, 3.369 $\pm 0.109 \mathrm{nmol}$ and SH-SY5Y cells, $2.233 \pm 0.036 \mathrm{nmol}$ ) (Fig. 2E). Furthermore, rotenone treatment increased ECAR compared to the basal level in U87MG cells $(28.294 \pm 1.107$ versus $21.842 \pm 1.446 \mathrm{nmol}$, respectively) (Fig. 2F). Density of cell in each well is important because the XF-analyzer measures the oxygen or proton in a small 

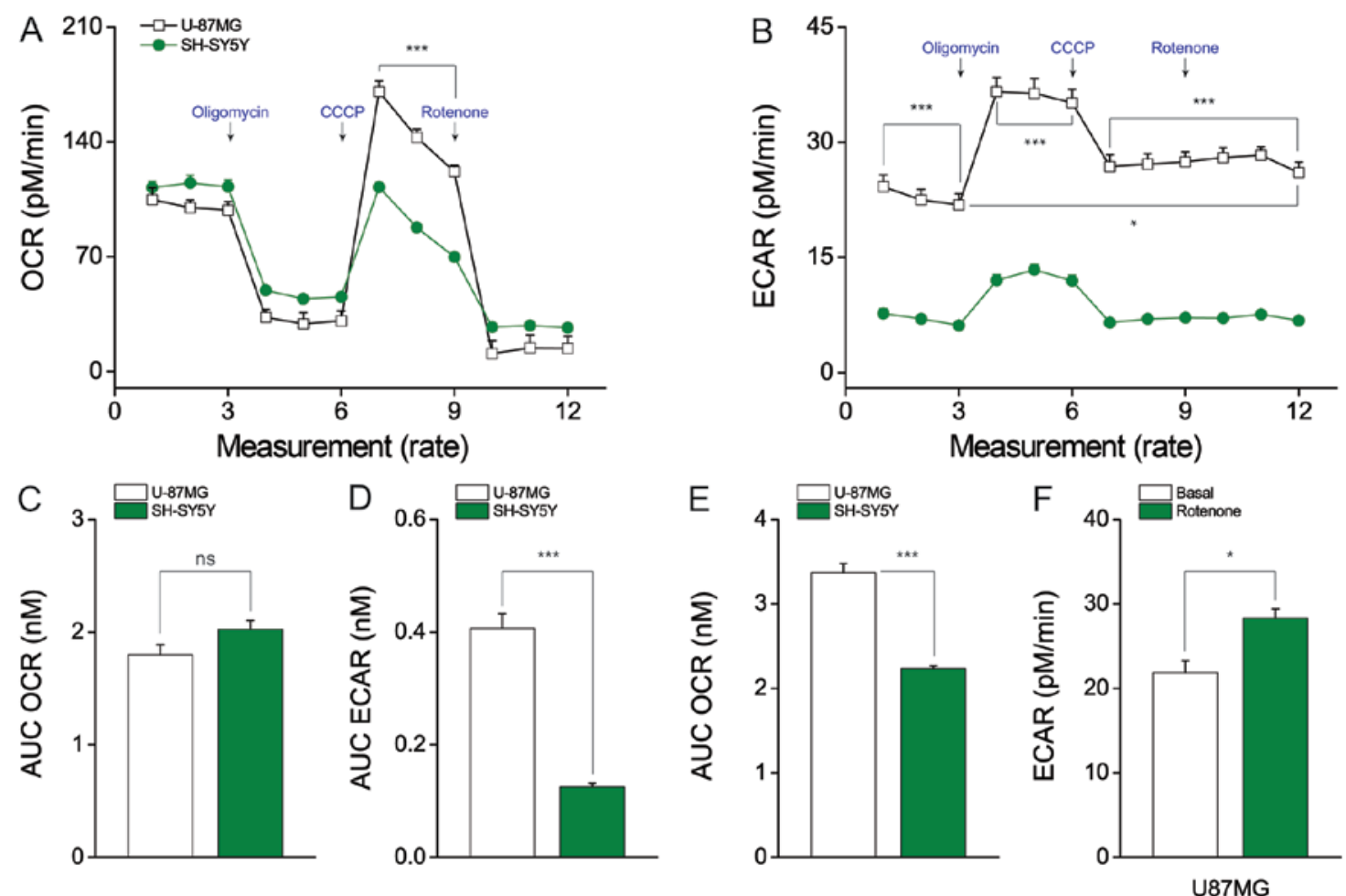

Figure 2. Lactate production differs between U87MG and SH-SY5Y cells, whereas mitochondrial function does not. (A) The oxygen consumption rate $(\mathrm{OCR})$, which indicates mitochondrial function, and (B) extracellular acidification rate (ECAR), which indicates lactate production, were analyzed with a Seahorse XF24 analyzer in cells treated with $2 \mu \mathrm{g} / \mathrm{ml}$ oligomycin (an ATPase inhibitor), $5 \mu \mathrm{M} \mathrm{CCCP}$ (an uncoupler), or $2 \mu \mathrm{M}$ rotenone (a mitochondrial complex I inhibitor). The AUC (area under the curve) of (C) basal OCR and (D) basal ECAR were measured from the first to third time-points. (E) The AUC of the maximal OCR was measured from the seventh to ninth time-points. (F) U87MG cells showed increased ECAR after rotenone treatment, compared with the basal ECAR. Data represent the means \pm SEM (error bars) from three experiments (U87MG, $n=10$; and SH-SY5Y, $n=10$ ). NS, not significant; ${ }^{*} \mathrm{P}<0.05$; and ${ }^{* * *} \mathrm{P}<0.001$ (by one-way ANOVA) compared with SH-SY5Y cells (A-E) and basal ECAR compared with rotenone-treated ECAR condition (F).


Figure 3. The mRNA expression profiles of metabolism-related genes in U87MG and SH-SY5Y cells. The mRNA expression levels of (A) $L D H-A$ (lactate dehydrogenase), (B) PC (pyruvate carboxylase), (C) SDH (succinate dehydrogenase), and (D) PPAR- $\gamma$ (peroxisome proliferator-activated receptor- $\gamma$ ) were analyzed in U87MG and SH-SY5Y cells using real-time PCR. Data represent the means \pm SEM (error bars) from three experiments (U87MG, $\mathrm{n}=5$; and SH-SY5Y, $n=5$ ). ${ }^{* *} \mathrm{P}<0.01$; and ${ }^{* * *} \mathrm{P}<0.001$ (by 2-tailed unpaired Student's t-test) compared with SH-SY5Y cells.

area in the middle of the well. Consequently, we seeded $95 \%$ of the area in the well with U87MG and SH-SY5Y cells, respectively. These results indicate potential mitochondrial function revealing high capacity in the U87MG cells in addition to glycolytic metabolic capacity. Consistent with this, we observed high-level expression of the gene encoding succinate dehydrogenase (SDH), which is involved in the TCA (Fig. 3C).

U87MG cells favor aerobic glycolysis at the transcriptional level. Lactate is a critical substrate for supplying energy in cancer (via the Warburg effect), and is believed to help determine low pH during carcinogenesis (21). Furthermore, the activation of aerobic glycolysis can trigger lactate production, and as a byproduct, it can also supply nicotinamide adenine dinucleotide (NAD) ${ }^{+}$as a co-substrate for glycolysis which induce rapid cancer cell growth (22). Here, we first examined the mRNA expression of LDH-A, which is the main enzyme responsible for lactate production. Consistent with the ECAR results, the level of LDH-A was $\sim 3$-fold higher in U87MG cells compared to SH-SY5Y cells (Fig. 3A). The mRNA expression 
A
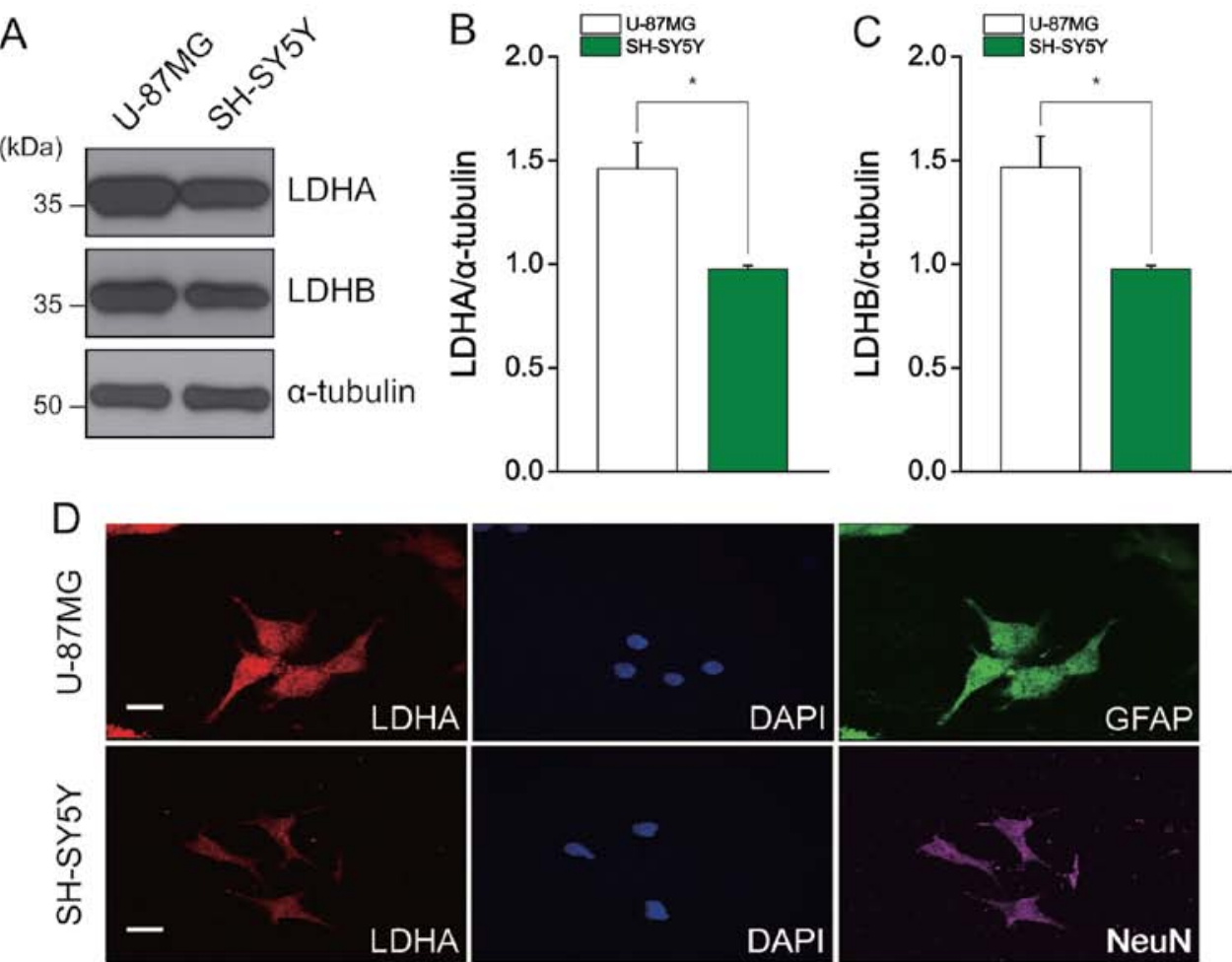

\section{$E$
$\sum_{\substack{\infty \\ j \\ J}}$}
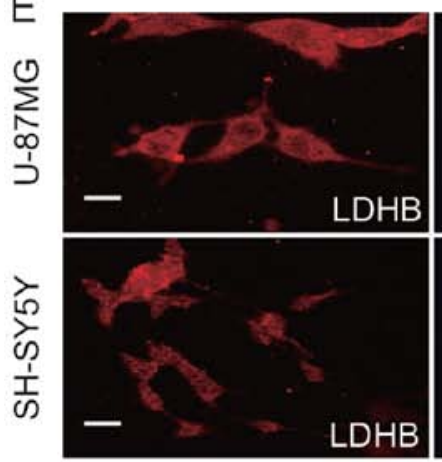
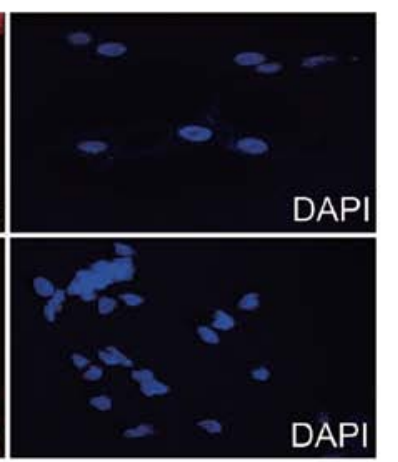
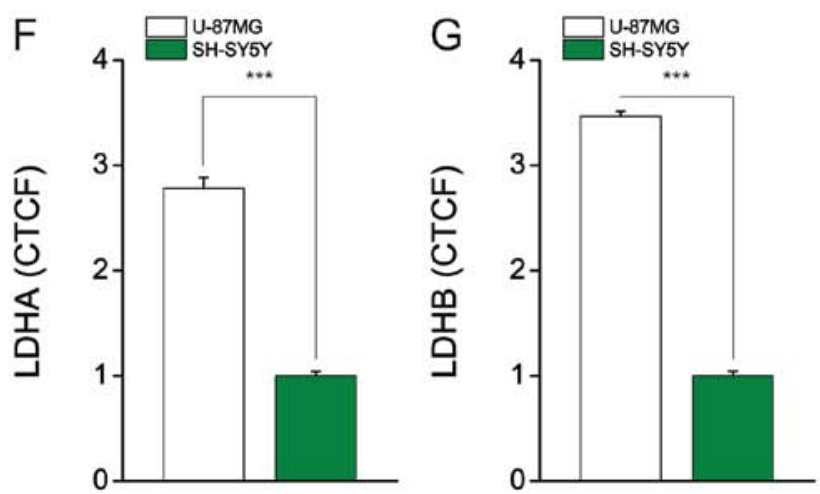

Figure 4. LDH-A and LDH-B protein expression levels in U87MG and SH-SY5Y cells. (A) The protein expression levels of LDH-A and LDH-B in U87MG and SH-SY5Y cells were analyzed by western blot analysis. The signal intensities of (B) LDH-A and (C) LDH-B were normalized with respect to that of $\alpha$-tubulin (D) U87MG and SH-SY5Y cells were stained by anti-GFAP (an astrocytic marker), anti-NeuN (a neuronal marker), anti-LDH-A and (E) anti-LDH-B with DAPI (a DNA marker), respectively. Measured intensities of (F) LDH-A and (G) LDH-B represent by value of CTCF (corrected total cell fluorescence). Data were normalized with level of intensities in SH-SY5Y cells that were stained LDH-A and LDH-B, respectively. Data represent the means \pm SEM (error bars) from three experiments [(B, C, F and G) U87MG, $\mathrm{n}=4$; and SH-SY5Y, n=4)]. ${ }^{*} \mathrm{P}<0.05$; and ${ }^{* * *} \mathrm{P}<0.001$ (2-tailed unpaired Student's t-test) compared with SH-SY5Y cells. The signals were assessed using confocal microscopy (x600). Scale bars, $200 \mu \mathrm{m}$.

level of pyruvate carboxylase (PC), which mediates gluconeogenesis (23), was relatively low in U87MG cells, supporting the use of pyruvate to produce lactate in GBM cells (Fig. 3B). Thus, the metabolic flow appears to tend toward aerobic glycolysis, which encourages fast growth. Moreover, the gene encoding proliferator-activated receptor-gamma $(P P A R-\gamma)$, which is involved in the production of palmitate (a component of the cellular membrane) and supports cellular division and growth, was induced in U87MG cells (Fig. 3D).

LDH protein expression is high in U87MG cells. The LDH-A and LDH-B subunits catalyze the forward and reverse reactions, respectively, between pyruvate and lactate. To assess the protein expression levels of LDH-A and LDH-B, we performed immunoblotting using specific antibodies (Fig. 4A). Consistent with the results of our mRNA expression analysis, the protein expression level of total LDH was $~ 1.5$-fold higher in U87MG cells compared to SH-SY5Y cells (Fig. 4B and C). Furthermore, immunofluorescent staining showed that expressional levels of LDH-A and B were consistent with our immunoblotting results (Fig. 4D and E). As U87MG and SH-SY5Y cells are derived from astrocytes and neurons, respectively, we stained these cells for GFAP (an astrocytic marker) and NeuN (a neuronal marker) to examine remnant cellular characteristics (Fig. 4D). Interestingly, those cells have expression of GFAP and NeuN, respectively, and U87MG cells which contain high expression of LDH seems to derive from original cellular peculiarity, the astrocyte. The levels of intensity with LDH-A and LDH-B were over 2.5-fold and 3-fold higher in U87MG cells compared to SH-SY5Y cells, respectively (Fig. 4F and G). The date were 
A

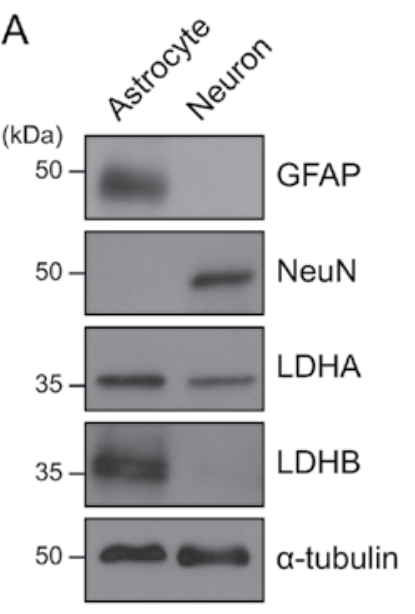

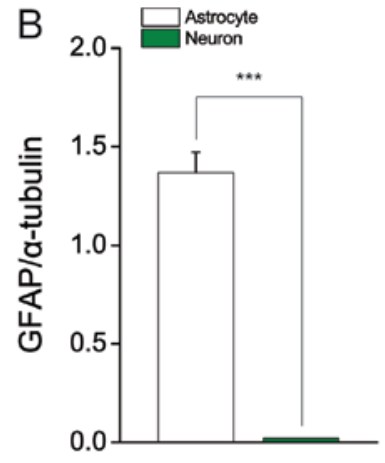

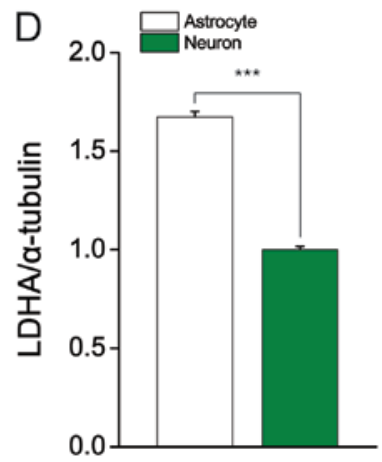

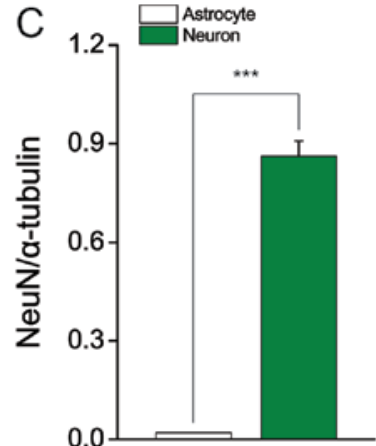

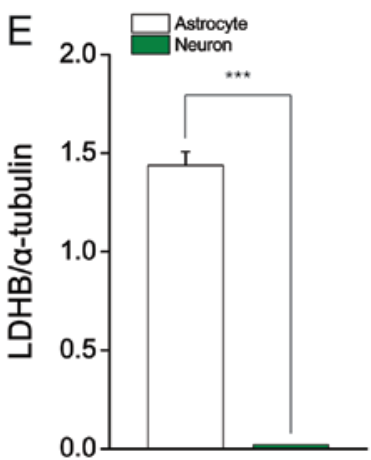

Figure 5. Innate characteristics of primary cultured astrocytes and neurons. (A) Western blotting was used to examine protein expression in primary cultured astrocytes and neurons. The signal intensity values of (B) GFAP, (C) NeuN, (D) LDH-A and (E) LDH-B were normalized with respect to that of $\alpha$-tubulin. Data represent the means \pm SEM (error bars) from three experiments [(B-E) astrocytes, $n=4$; and neurons, $n=4)] .{ }^{* * *} \mathrm{P}<0.001$ (2-tailed unpaired Student's $t$-test) compared with neurons.

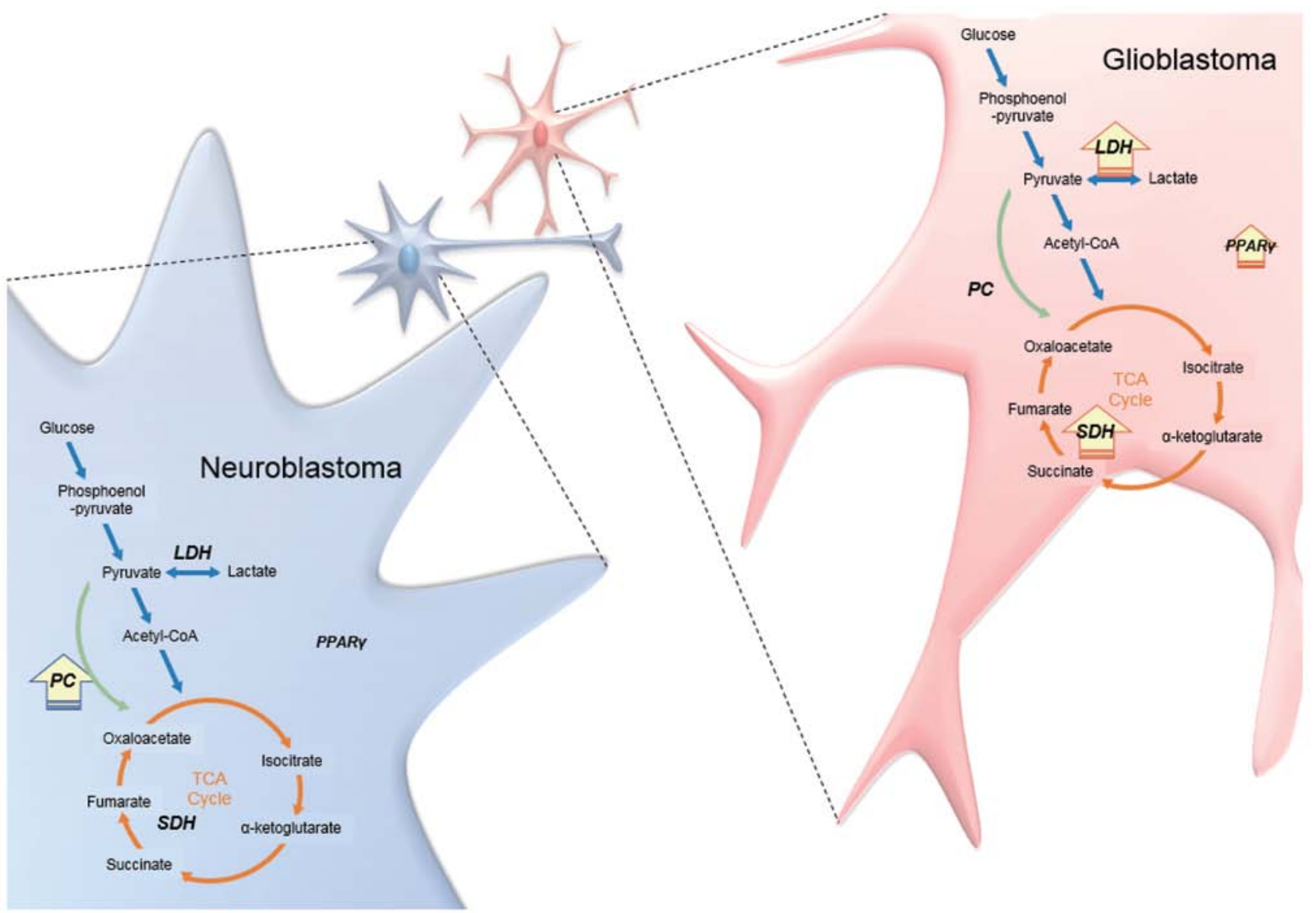

Figure 6. Metabolism of astrocyte-derived GBM cells and neuron-derived NB cells. Schematic of the different metabolic pathways in GBM and NB. The arrows indicate: i) glycolysis (blue); ii) the TCA cycle (orange) for glucose oxidation; iii) PC (green) which is a pathway of anaplerosis, a process that replenishes TCA cycle intermediates; and iv) PPAR- $\gamma$. LDH, lactate dehydrogenase; SDH, succinate dehydrogenase; PC, pyruvate carboxylase; and PPAR- $\gamma$, peroxisome proliferator-activated receptor- $\gamma$. 
processed by the corrected total cell fluorescence (CTCF). The value of CTCF indicates that integrated density without measured fluorescence by background.

LDH protein expression is high in primary cultured cortical astrocytes. Astrocytes and neurons have different metabolic profiles, allowing them to carry out their specific roles in the brain. Lactate production and glycogen storage tend to be higher in astrocytes than neurons (24). Here, we evaluated $\mathrm{LDH}$ expression in the astrocytes and neurons of primary cultures (Fig. 5A). The markers for astrocytes (GFAP) and neurons $(\mathrm{NeuN})$ were only expressed by astrocytes and neurons, respectively (Fig. 5B and C). As expected, the expression levels of LDH-A and LDH-B were higher in astrocytes than in neurons (Fig. 5D and E). These results may suggest that the increased lactate production of astrocytes is maintained in GBM cells, supporting high-level cellular growth by fulfilling intracellular ATP demands.

\section{Discussion}

Cancer cells require a particular environment to flourish, including the acidic conditions that arise from the production of lactate via aerobic glycolysis (25). Furthermore, lactate inhibits the T-cell response and interrupts the immune system in cancer cells (26). Thus, the proliferation of cancer cells can be regulated by restricting lactate production (27). Here, we present evidence suggesting that the high proliferative capacity of GBM cells is related to the innate metabolic phenotype of astrocytes, which tends toward lactate production.

Uncontrolled proliferation of tumor cells is an obstacle for devising therapeutic strategy in GBM patients with temozolomide (common drug of GBM). In several patients, GBM cells have been shown to resist temozolomide treatment through the repair of DNA damages (28). In an effort to identify a therapeutic target with prominent effects on the proliferation of GBM cells, we focused on the metabolic organization related to GBM. Under conditions of unrestricted oxygen, normal cells process energy via the Pasteur effect, with mitochondrial respiration for producing ATP at high efficiency (29). In cancer cells, however, the metabolic organization is altered to favor the Warburg effect, wherein ATP preferentially is produced by glycolysis flux with low efficiency of mitochondrial oxidative phosphorylation and lactate is generated, even in the presence of a sufficient oxygen supply (17). Interestingly, astrocytes have higher metabolic capacity than neuron and contribute to metabolic support to neurons through production of lactate, concomitantly, astrocyte produce ATP in mitochondria for self-energy needs. Consistent with this, we found that U87MG cells simultaneously had higher ECAR, higher potential mitochondrial function, and higher growth ability compared to SH-SY5Y cells. Moreover, ECAR remained relatively high in U87MG cells following rotenone treatment, and cultured astrocytes had higher LDH expression than neurons in our system. Together, these findings indicate that, similar to astrocytes, U87MG cells favor lactate production.

A previous transcriptional profiling showed that other TCA cycle enzymes, including citrate synthase and malate dehydrogenase, were more highly expressed in astrocytes than in neurons (30). Here, we noted that succinate dehydrogenase was also expressed at a relatively higher level in GBM cells compared to SH-SY5Y cells (Fig. 3C). The production of a co-substrate capable of activating the mitochondrial respiratory chain from the TCA cycle (e.g., FADH or NADH) could increase cell division and proliferation, as seen in adenocarcinoma originating from a primary unknown metastatic cancer (31). Moreover, we noted higher PPAR- $\gamma$ expression in GBM cells, which could contribute to their rapid proliferation. Consistent with this, a previous study found that PPAR- $\gamma$ activation induced tumor formation and reduced the overall survival time in pancreatic cancer (32). GBM cells have highlevel of $L D H$ as glycolytic enzyme and $S D H$ as enzyme for TCA cycle compared to NB cells. Expression of PPAR- $\gamma$ was elevated and is capable of contribution to cellular membrane formation rather than NB cells. Otherwise, NB cells contain high-level of PC for energy production with transported lactate from astrocyte compared to GBM cells (Fig. 6).

The polarity of the metabolic organization that support the rapid growth of GBM cells appears to be related to the upregulation of both glycolysis- and TCA cycle-related enzymes. Here, we report that U87MG cells have a metabolic phenotype similar to that of astrocytes, but mitochondrial respiration does not appear to be different between U87MG and SH-SY5Y cells. The determining factor for rapid growth appears to be a difference in lactate dehydrogenase expression in GBM cells, leading to a higher ECAR. According to recent reports, inhibition of LDH-A has anticancer effect by restriction of energy in human lymphoma and pancreatic xenograft tumor growth $(33,34)$. Furthermore, inhibition of LDH-A suppresses proliferation in separated cells from mammary gland tumor (35). Based on these findings, we suggest that the inherent peculiarity of astrocytic metabolism, wherein the Pasteur and Warburg effects are both at work, facilitates glioblastoma. This new understanding of the metabolic characteristics of brain tumor cells could help guide the future development of novel targeted drugs.

\section{Acknowledgements}

Primary culture of astrocytes and neurons received help from the laboratory of C. Justin Lee in Korea Institute of Science and Technology. This study was supported by the National Research Foundation of Korea (NRF) grant funded by the Ministry of Science, ICT \& Future Planning (MSIP) (2014R1A2A1A11051231), (2014R1A1A1037655) and by the Ministry of Education (2014R1A6A1029617) and research fund of Chungnam National University.

\section{References}

1. Ohgaki $\mathrm{H}$ and Kleihues P: Population-based studies on incidence, survival rates, and genetic alterations in astrocytic and oligodendroglial gliomas. J Neuropathol Exp Neurol 64: 479-489, 2005.

2. Campbell AM, Rennie JS, Moos KF and Patton D: Neuroblastoma presenting as mandibular swelling in a two-year-old girl - a short case report. Br J Oral Maxillofac Surg 25: 422-426, 1987.

3. Diehn M, Nardini C, Wang DS, McGovern S, Jayaraman M Liang Y, Aldape K, Cha S and Kuo MD: Identification of noninvasive imaging surrogates for brain tumor gene-expression modules. Proc Natl Acad Sci USA 105: 5213-5218, 2008.

4. Buckner JC: Factors influencing survival in high-grade gliomas. Semin Oncol 30 (Suppl 19): S10-S14, 2003. 
5. Bouzier-Sore AK and Pellerin L: Unraveling the complex metabolic nature of astrocytes. Front Cell Neurosci 7: 179, 2013.

6. Bélanger M, Allaman I and Magistretti PJ: Brain energy metabolism: Focus on astrocyte-neuron metabolic cooperation. Cell Metab 14: 724-738, 2011

7. Suzuki A, Stern SA, Bozdagi O, Huntley GW, Walker RH, Magistretti PJ and Alberini CM: Astrocyte-neuron lactate transport is required for long-term memory formation. Cell 144 810-823, 2011.

8. Walz W and Mukerji S: Lactate production and release in cultured astrocytes. Neurosci Lett 86: 296-300, 1988.

9. Dringen R, Wiesinger H and Hamprecht B: Uptake of L-lactate by cultured rat brain neurons. Neurosci Lett 163: 5-7, 1993.

10. Bagley PR, Tucker SP, Nolan C, Lindsay JG, Davies A Baldwin SA, Cremer JE and Cunningham VJ: Anatomical mapping of glucose transporter protein and pyruvate dehydrogenase in rat brain: An immunogold study. Brain Res 499: 214-224, 1989.

11. Cahn RD, Zwilling E, Kaplan NO and Levine L: Nature and Development of Lactic Dehydrogenases: The two major types of this enzyme form molecular hybrids which change in makeup during development. Science 136: 962-969, 1962.

12. Bittar PG, Charnay Y, Pellerin L, Bouras C and Magistretti PJ: Selective distribution of lactate dehydrogenase isoenzymes in neurons and astrocytes of human brain. J Cereb Blood Flow Metab 16: 1079-1089, 1996.

13. Crane CA, Austgen K, Haberthur K, Hofmann C, Moyes KW, Avanesyan L, Fong L, Campbell MJ, Cooper S, Oakes SA, et al: Immune evasion mediated by tumor-derived lactate dehydrogenase induction of NKG2D ligands on myeloid cells in glioblastoma patients. Proc Natl Acad Sci USA 111: 12823-12828, 2014.

14. Nieder C, Marienhagen K, Dalhaug A, Aandahl G, Haukland E and Pawinski A: Prognostic models predicting survival of patients with brain metastases: Integration of lactate dehydrogenase, albumin and extracranial organ involvement. Clin Oncol (R Coll Radiol) 26: 447-452, 2014.

15. Woo DH, Han KS, Shim JW, Yoon BE, Kim E, Bae JY, Oh SJ, Hwang EM, Marmorstein AD, Bae YC, et al: TREK-1 and Best1 channels mediate fast and slow glutamate release in astrocytes upon GPCR activation. Cell 151: 25-40, 2012.

16. Biedler JL, Helson L and Spengler BA: Morphology and growth, tumorigenicity, and cytogenetics of human neuroblastoma cells in continuous culture. Cancer Res 33: 2643-2652, 1973.

17. Warburg O: On respiratory impairment in cancer cells. Science 124: 269-270, 1956

18. Vander Heiden MG, Cantley LC and Thompson CB: Understanding the Warburg effect: The metabolic requirements of cell proliferation. Science 324: 1029-1033, 2009.

19. Wang R, Novick SJ, Mangum JB, Queen K, Ferrick DA, Rogers GW and Stimmel JB: The acute extracellular flux (XF) assay to assess compound effects on mitochondrial function. J Biomol Screen 20: 422-429 2015.
20. Park JW, Lee SY, Yang JY, Rho HW, Park BH, Lim SN, Kim JS and Kim HR: Effect of carbonyl cyanide m-chlorophenylhydrazone (CCCP) on the dimerization of lipoprotein lipase. Biochim Biophys Acta 1344: 132-138, 1997.

21. Xie J, Wu H, Dai C, Pan Q, Ding Z, Hu D, Ji B, Luo Y and Hu X: Beyond Warburg effect - dual metabolic nature of cancer cells. Sci Rep 4: 4927, 2014.

22. Chiarugi A, Dölle C, Felici R and Ziegler M: The NAD metabolome - a key determinant of cancer cell biology. Nat Rev Cancer 12: 741-752, 2012.

23. Jitrapakdee S, St Maurice M, Rayment I, Cleland WW, Wallace JC and Attwood PV: Structure, mechanism and regulation of pyruvate carboxylase. Biochem J 413: 369-387, 2008.

24. Brown AM and Ransom BR: Astrocyte glycogen and brain energy metabolism. Glia 55: 1263-1271, 2007.

25. Gatenby RA and Gillies RJ: Why do cancers have high aerobic glycolysis? Nat Rev Cancer 4: 891-899, 2004.

26. Fischer K, Hoffmann P, Voelkl S, Meidenbauer N, Ammer J, Edinger M, Gottfried E, Schwarz S, Rothe G, Hoves S, et al: Inhibitory effect of tumor cell-derived lactic acid on human T cells. Blood 109: 3812-3819, 2007.

27. Hirschhaeuser F, Sattler UG and Mueller-Klieser W: Lactate: A metabolic key player in cancer. Cancer Res 71: 6921-6925, 2011.

28. Van Meir EG, Hadjipanayis CG, Norden AD, Shu HK, Wen PY and Olson JJ: Exciting new advances in neuro-oncology: The avenue to a cure for malignant glioma. CA Cancer J Clin 60: 166-193, 2010.

29. Stickland LH: The Pasteur effect in normal yeast and its inhibition by various agents. Biochem J 64: 503-515, 1956.

30. Lovatt D, Sonnewald U, Waagepetersen HS, Schousboe A, He W, Lin JH, Han X, Takano T, Wang S, Sim FJ, et al: The transcriptome and metabolic gene signature of protoplasmic astrocytes in the adult murine cortex. J Neurosci 27: 12255-12266, 2007.

31. Kim HM, Kim H, Jung WH and Koo JS: Metabolic phenotypes in primary unknown metastatic carcinoma. J Transl Med 12: 2, 2014.

32. Nakajima A, Tomimoto A, Fujita K, Sugiyama M, Takahashi H, Ikeda I, Hosono K, Endo H, Yoneda K, Iida H, et al: Inhibition of peroxisome proliferator-activated receptor gamma activity suppresses pancreatic cancer cell motility. Cancer Sci 99: 1892-1900, 2008

33. Omar HA, Berman-Booty L, Kulp SK and Chen CS: Energy restriction as an antitumor target. Future Oncol 6: 1675-1679, 2010.

34. Le A, Cooper CR, Gouw AM, Dinavahi R, Maitra A, Deck LM, Royer RE, Vander Jagt DL, Semenza GL and Dang CV: Inhibition of lactate dehydrogenase A induces oxidative stress and inhibits tumor progression. Proc Natl Acad Sci USA 107: 2037-2042, 2010.

35. Fantin VR, St-Pierre J and Leder P: Attenuation of LDH-A expression uncovers a link between glycolysis, mitochondrial physiology, and tumor maintenance. Cancer Cell 9: 425-434, 2006. 